\title{
Die Regelung der Suizidbeihilfe in den neuen SAMW-Richtlinien
}

\section{Michael Barnikol}

Dr. iur., Jurist im Rechtsdienst der FMH, Mitglied der Einsprachekommissionen Weiterbildungsstätten und Weiterbildungstitel

Die Schweizerische Akademie der Medizinischen Wissenschaften (SAMW) hat ihre Richtlinien «Betreuung von Patientinnen und Patienten am Lebensende» einer Totalrevision unterzogen. Die neuen Richtlinien mit dem Titel «Umgang mit Sterben und Tod» wurden am 6. Juni 2018 veröffentlicht. Sie enthalten eine bedeutsame Änderung der Regelung, unter welchen Voraussetzungen Ärztinnen und Ärzte Suizidbeihilfe leisten dürfen.

Die alte Regelung der Suizidbeihilfe (Ziff. 4.1 der Richtlinien «Betreuung von Patientinnen und Patienten am Lebensende») und die neue Regelung (Ziff. 6.2.1 der Richtlinien «Umgang mit Sterben und Tod») enthalten viele Gemeinsamkeiten und sind gleich strukturiert. Beide Regelungen halten fest, dass die Rolle des Arztes darin besteht, «Symptome zu lindern und den Patienten zu begleiten». Es gehöre demnach «weder zu seinen Aufgaben, von sich aus Suizidhilfe anzubieten, noch [sei] er verpflichtet, diese zu leisten». Suizidbeihilfe sei «keine medizinische Handlung, auf die Patientinnen und Patienten einen Anspruch erheben könnten $[\ldots] »$.

Unter bestimmten Voraussetzungen soll es einem Arzt oder einer Ärztin, die mit einem Suizidwunsch konfrontiert werden, allerdings erlaubt sein, Suizidbeihilfe zu leisten. Die Abklärung, ob diese Voraussetzungen vorliegen, legen die Richtlinien in die Hände des Suizidbeihilfe leistenden Arztes und einer unabhängigen Drittperson. Die Leistung von Suizidbeihilfe stellt gemäss Ziff. 6.2.1 der Richtlinien demgemäss einen "persönlich verantworteten Entscheid" des Arztes dar. Die wichtigste Voraussetzung für die Leistung von Suizidbeihilfe ist sowohl in der alten als auch in der neuen Regelung die Urteilsfähigkeit. Dies ist gemäss Art. 16 des Schweizerischen Zivilgesetzbuchs die Fähigkeit zu vernunftgemässem Handeln. Das Fehlen der Urteilsunfähigkeit des Patienten oder der Patientin muss sorgfältig dokumentiert werden. Wenn ein Zustand des Patienten vorliegt, der häufig mit fehlender Urteilsfähigkeit verbunden ist Ziff. 6.2.1 der Richtlinien nennt diesbezüglich Demenz und psychische Krankheiten -, ist die Urteilsfähigkeit von einem sachkundigen Facharzt zu evaluieren. Der
Suizidwunsch muss zudem "wohlerwogen und ohne äusseren Druck entstanden sowie dauerhaft» sein. Ziff. 6.2.1 der Richtlinien verlangt des Weiteren, dass «medizinisch indizierte therapeutische Optionen sowie andere Hilfs- und Unterstützungsangebote gesucht [wurden] und erfolglos geblieben [sind] oder vom diesbezüglich urteilsfähigen Patienten als unzumutbar abgelehnt [werden]». Der Suizidwunsch muss «für den Arzt aufgrund der Vorgeschichte und wiederholter Gespräche nachvollziehbar» und es muss «für ihn vertretbar [sein], in diesem konkreten Fall Suizidhilfe zu leisten".

In einem Punkt unterscheiden sich die neuen Richtlinien wesentlich von den alten. Dieser betrifft den gesundheitlichen Zustand des Patienten oder der Patientin: Während die alten Richtlinien Suizidbeihilfe nur

Die Revision der Richtlinien weitet die Anwendbarkeit von Suizidbeihilfe in nicht unerheblichem Masse aus.

dann erlauben, wenn «die Erkrankung des Patienten die Annahme [rechtfertigt], dass das Lebensende nahe ist», ist Suizidbeihilfe gestützt auf Ziff. 6.2.1 der neuen Richtlinien bereits dann zulässig, wenn «die Krankheitssymptome und/oder Funktionseinschränkungen des Patienten für diesen Ursache unerträglichen Leidens» sind. Die Revision der Richtlinien weitet die Anwendbarkeit von Suizidbeihilfe somit in nicht unerheblichem Masse aus, woraus sich eine Reihe von juristischen und praktischen Fragestellungen ergeben. Bevor diese aufgezeigt werden, soll zunächst auf die Bedeutung der SAMW-Richtlinien für die Ärzteschaft eingegangen werden. 


\section{Bedeutung der SAMW-Richtlinien}

Obwohl den Richtlinien der SAMW keine Gesetzesqualität zukommt, haben sie im Gesundheitsrecht sowohl rechtlich als auch faktisch eine grosse Bedeutung [1]. Sie sind Teil des ärztlichen Standesrechts (Art. 18 der Standesordnung der FMH, nachfolgend: StaO). Ein Verstoss gegen die Richtlinien ist deshalb eine Verletzung ärztlichen Standesrechts und kann von den Standeskommissionen der kantonalen Ärztegesellschaften z.B. mit Geldbussen oder dem Ausschluss aus der Gesellschaft sanktioniert werden (Art. $47 \mathrm{StaO}$ ). Da die kantonalen Standeskommissionen keine Aufsichtsbehörden sind, werden sie allerdings nur dann aktiv, wenn das standeswidrige Verhalten bei ihnen angezeigt wird. Was die Regelung der Suizidbeihilfe betrifft, ist zu beachten, dass Art. 18 StaO gegenwärtig auf die alten Richtlinien "Betreuung von Patientinnen und Patienten am Lebensende» verweist. Damit die neuen Richtlinien Teil des ärztlichen Standesrechts werden, ist deshalb eine Änderung der Standesordnung nötig, was einen entsprechenden Beschluss der Ärztekammer der FMH voraussetzt.

\section{Obwohl den Richtlinien der SAMW keine Gesetzesqualität zukommt, haben sie eine grosse Bedeutung.}

Des Weiteren konkretisieren die Richtlinien den Sorgfaltsmassstab, dessen Einhaltung von Ärztinnen bzw. Ärzten verlangt werden darf, und zeigen den Normsetzungsbedarf in gesetzlich noch ungeregelten Gebieten auf [2]. Auch die bundesgerichtliche Rechtsprechung zieht die Richtlinien der SAMW als Entscheidungsmassstab bei und spricht ihnen eine hohe Legitimität $\mathrm{zu}$ [3]. Dies gilt in besonderem Masse für die Regelung der Suizidbeihilfe in den Richtlinien der SAMW. Da die Suizidbeihilfe in der Schweiz auf Bundesebene ausser im Strafrecht gesetzlich nicht geregelt ist, hat das Bundesgericht in seiner Rechtsprechung auf die entsprechende Regelung in den SAMW-Richtlinien verwiesen [4].

\section{Suizidprävention und Patientenautonomie}

Die Frage der Suizidbeihilfe ist von einem Spannungsverhältnis zwischen zwei ethischen Forderungen geprägt, nämlich einerseits derjenigen nach einer effektiven Suizidprävention und andererseits derjenigen nach der Aufrechterhaltung der Patientenautonomie [5]. Der Arzt oder die Ärztin sind gegenüber suizidgefährdeten Menschen zur Fürsorge verpflichtet - gleich- zeitig müssen sie aber auch das Selbstbestimmungsrecht der Patientinnen und Patienten berücksichtigen [5]. Auf juristischer Ebene bedarf es einer Regelung, die dem betroffenen Arzt oder der betroffenen Ärztin erlaubt, im Voraus sicher abschätzen zu können, ob die Voraussetzungen der Suizidbeihilfe gegeben sind. $\mathrm{Zu}-$ dem muss das Verhalten eines Arztes im Nachhinein gestützt auf klare Regeln zuverlässig überprüfbar sein

Eine Vorschrift darf nicht so unbestimmt sein, dass keine rechtssichere und rechtsgleiche Anwendung mehr möglich ist.

können. Die SAMW stand deshalb bei der Revision der Suizidbeihilferegelung vor einer grossen Herausforderung. Indem sie die Anwendbarkeit der Suizidbeihilfe nicht mehr auf das Lebensende beschränkte, wurde sie der gesellschaftlichen Realität gerecht, dass der Suizidwunsch von schwer erkrankten Patientinnen und Patienten oft bereits zu einem viel früheren Zeitpunkt geäussert wird. Die Ausweitung der Suizidbeihilfe eröffnet jedoch gleichzeitig eine Vielzahl neuer Fragen und Problemkreise.

\section{Unbestimmtheit des Kriteriums «unerträgliches Leiden»}

Diese Fragen resultieren in erster Linie daraus, dass der Begriff des «unerträglichen Leidens» sehr unbestimmt und zudem subjektiv geprägt ist. Die Regelung stellt nicht allein auf den Zustand des Patienten oder der Patientin ab, sondern primär darauf, wie der Patient diesen Zustand empfindet. Unbestimmte Rechtsbegriffe sind keineswegs ungewöhnlich und können konkretisiert werden, indem der Rechtsanwender diese auslegt und eine entsprechende Praxis bildet [6] Die Unbestimmtheit einer Norm darf jedoch nicht so weit gehen, dass keine rechtssichere und rechtsgleiche Anwendung der Bestimmung mehr möglich ist - dies besagt das aus dem Rechtsstaatsprinzip abzuleitende Bestimmtheitsgebot [7]. Auch die Bestimmungen der SAMW-Richtlinien verlangen nach einer rechtsgleichen und rechtssicheren Anwendung. Deshalb sollten sie, obschon ihnen keine Gesetzesqualität zukommt, zumindest im Wesentlichen den Anforderungen des Bestimmtheitsgebots genügen. Der Bestimmtheitsgrundsatz besagt, dass eine Vorschrift so präzise formuliert sein muss, dass der Bürger sein Verhalten danach einrichten und die Folgen eines bestimmten Verhaltens mit einem den Umständen entsprechenden Grad an Gewissheit erkennen kann [8]. Der erforderliche Grad der Bestimmtheit hängt unter anderem von 
der Vielfalt der zu ordnenden Sachverhalte, von der Komplexität und der Vorhersehbarkeit der im Einzelfall erforderlichen Entscheidungen, von den Normadressaten, von der Schwere des Eingriffs in Verfassungsrechte und von der erst bei der Konkretisierung im Einzelfall möglichen und sachgerechten Entscheidung $\mathrm{ab}$ [9].

Die Situationen, in denen ein Patient oder eine Patientin einen Arzt um Beihilfe zum Suizid bitten können, sind komplex und können sehr unterschiedlich sein. So ist beispielsweise der Fall eines schwer depressiven Patienten anders gelagert, als der eines Patienten, bei dem Krebs im Endstadium diagnostiziert worden ist. Die Entscheidung des Arztes oder der Ärztin für oder gegen Suizidbeihilfe betrifft zudem das Leben und das Selbstbestimmungsrecht des Patienten - es geht also um schwerwiegende Entscheidungen mit weitreichenden Konsequenzen für besonders schützenswerte Rechtsgüter. Es ist deshalb ein sehr hoher Grad an Bestimmtheit zu fordern. Ob die Regelung in Ziff. 6.2.1 diesen Anforderungen gerecht wird, erscheint fraglich. Eine sichere Grenzziehung zwischen noch erträglichem und unerträglichem Leiden in einer Weise, die eine zuverlässige und nachvollziehbare rechtliche Überprüfung ermöglicht, ist aus mehreren Gründen, die nachfolgend aufgezeigt werden sollen, kaum gewährleistet.

\section{Beurteilung des Leidens einer anderen Person}

Die beschriebene Abgrenzungsschwierigkeit ergibt sich nicht nur aus der äusserst vagen Formulierung des Begriffs «unerträgliches Leiden», sondern auch daraus, dass die neue Suizidbeihilferegelung vom Arzt oder der Ärztin verlangt, das Leiden einer anderen Person

Ein «intersubjektiver Nachvollzug» des Leidens durch den Arzt oder die Ärztin ist kaum überprüfbar und bietet keine Rechtssicherheit.

zu beurteilen und zu qualifizieren. Die Verantwortung für den Entscheid, Suizidbeihilfe zu leisten, liegt, wie eingangs erwähnt, allein beim Arzt oder der Ärztin der Patient trägt demgegenüber die Verantwortung für die Durchführung des Suizids. Dies entspricht dem Wortlaut der Regelung und zudem dem Grundsatz, dass jede Person für ihre eigenen Handlungen verantwortlich ist. Gemäss Ziff. 6.2.1 der Richtlinien ist es deshalb Sache des Arztes, die Frage, ob der Patient unerträglich leidet, eindeutig und abschliessend $\mathrm{zu}$ beantworten. Dies stellt den betroffenen Arzt vor ein
Dilemma, weil es einerseits ihm obliegt, die Voraussetzungen der Suizidbeihilferegelung zu prüfen, andererseits aber das Leiden «für den Patienten» unerträglich sein muss - es ist mit anderen Worten aus der Perspektive des Patienten und nicht des Arztes zu beurteilen. Ein befriedigender Ausweg aus diesem Dilemma ist kaum möglich: Es kann nicht Aufgabe des Arztes sein, den Patienten zu bevormunden, indem er seine eigene Bewertung, ob das Leiden des Patienten unerträglich ist, an die Stelle der Einschätzung des Patienten setzt. Es wäre jedoch ebenso verfehlt, allein die Behauptung des Patienten, sein Leiden sei unerträglich, genügen zu lassen, denn die Verantwortung für die Beihilfe trägt letztlich nicht der Patient oder die Patientin, sondern die Ärztin oder der Arzt. Da der Suizidwunsch regelmässig daraus resultiert, dass der Patient seine Situation als unerträglich empfindet, würde dies zudem dazu führen, dass das Kriterium des unerträglichen Leidens bei Patienten, die einen Sterbewunsch äussern, faktisch so gut wie immer erfüllt wäre und damit seine praktische Bedeutung verlieren würde.

\section{Lösungsansatz der SAMW-Richtlinien}

Die SAMW vertritt in Abschnitt 6.2 der Richtlinien die Auffassung, dass die Unerträglichkeit des Leidens «im intersubjektiven Nachvollzug durch die behandelnde Ärztin plausibel gemacht werden» könne. Dies solle neben dem Vorliegen "medizinisch fassbarer Krankheitssymptome oder Funktionseinschränkungen" genügen. Es ist indessen zu bezweifeln, dass ein solcher Nachvollzug - in welcher Form dieser zu erfolgen hat, lassen die Richtlinien offen [10] - eine rechtsgleiche und rechtssichere Anwendung der Vorschrift ermöglicht, die den hohen Anforderungen an die Bestimmtheit der Norm gerecht wird. Die Intensität des Leidens des Patienten, die Weise, wie der Patient dieses empfindet, und die Massstäbe, die den Patienten bei der Bewertung dieses Leidens leiten, sind keine messbaren oder rechtlich zuverlässig überprüfbaren Kriterien. $\mathrm{Ob}$ jemand sein Leiden als erträglich empfindet, hängt von einer Vielzahl von nur schwer fassbaren Faktoren $\mathrm{ab}, \mathrm{zu}$ denen beispielsweise materielle, soziale oder psychische Ressourcen und die sozialen Bedingungen der Patientin oder des Patienten zählen [11]. Man muss sich in diesem Zusammenhang auch vor Augen halten, dass die Voraussetzungen der Suizidbeihilfe nicht nur für den Suizidbeihilfe leistenden Arzt nachvollziehbar und überprüfbar sein müssen, sondern auch für die Instanz, die beurteilt, ob das Verhalten des Arztes der Richtlinie entspricht (z.B. eine kantonale Standeskommission). Letztere kann das subjektiv empfundene Lei- 
den des Patienten noch weniger nachvollziehen als der behandelnde Arzt, zu dem der Patient ein Vertrauensverhältnis hat. Des Weiteren ist nicht ersichtlich, welche Methoden und Kompetenzen einen Arzt befähigen könnten, ein Patientenleiden intersubjektiv nachzuvollziehen [12].

\section{Psychische Krankheiten und Suizidprävention}

Ferner ist zu beachten, dass die Formulierung der neuen Richtlinie eine kaum überschaubare Anzahl an Krankheitsbildern erfasst - alle medizinisch fassbaren Krankheitssymptome oder Funktionseinschränkungen sollen Grundlage für ein «unerträgliches Leiden» sein können [13]. Die neue Regelung verlangt keine bestimmte Art oder Schwere der Erkrankung und dürfte folglich auch dann anwendbar sein, wenn die Krankheit des Patienten oder der Patientin therapierbar oder

\section{Die neue Regelung verlangt keine bestimmte Art oder Schwere der Erkrankung.}

heilbar ist. Die neue Suizidbeihilferegelung wird damit insbesondere auch auf solche Fälle anwendbar, in denen der Suizidwunsch durch die Krankheit beeinflusst oder sogar hervorgerufen wird und die Erkrankung Einfluss auf die Willensbildung des Patienten oder der Patientin haben kann. Dies betrifft insbesondere Patienten mit psychischen Erkrankungen wie Depression oder Demenz. Diese Patienten sind nicht zwingend urteilsunfähig [14], weshalb die Zulässigkeit der Suizidbeihilfe nicht schon an deren fehlender Urteilsfähigkeit scheitern muss. So kann beispielsweise selbst bei einer mittelschweren Demenz unter Umständen noch von der Urteilsfähigkeit des Patienten ausgegangen werden [15]. Dennoch ist anzunehmen, dass in diesen Fällen die Erkrankung einen Einfluss auf die freie Willensbildung und die Entstehung des Suizidwunsches des Patienten hat. Hierfür spricht etwa, dass in Ländern mit hohen Einkommen psychische Erkrankungen bei bis zu 90\% der Menschen vorhanden sind, die durch Suizid sterben [16].

Die Problematik verschärft sich dann zusätzlich, wenn das Leiden gut therapierbar oder - wie im Fall der Depression - auf die Dauer regelmässig sogar heilbar ist. Hier tritt die ärztliche Pflicht, Krankheiten zu behandeln bzw. zu heilen und Leiden zu lindern (vgl. Art. 2 StaO), in Konkurrenz zum Suizidbegehren des Patienten [17], und es stellt sich die Frage, ob die sehr weitgehende Ausweitung der Regelung einer effektiven Suizidprävention entgegenwirkt, die neben der Gewährleistung der Patientenautonomie den zweiten wichtigen Eckpunkt einer ausgewogenen Regelung der Suizidbeihilfe bilden sollte.

Das Bundesgericht führt diesbezüglich aus, Erkenntnisse der Suizidforschung und die Erfahrungen von Fachpersonen zeigten, «dass der Suizidwunsch regelmässig Ausdruck einer existentiellen Krisensituation» sei und «kaum Zeugnis eines in sich abgeklärten und gefestigten Willens» [18]. Bekannt sei zudem «die Labilität des Todeswunsches, gerade auch bei Schwerkranken» [18]. Zudem scheine «das Sterben-Wollen wesentlich von Schmerzen, von depressiven Symptomen und der erlebten Qualität der Pflege abhängig zu sein, aber auch von der Furcht, im Stich gelassen zu werden und anderen zur Last zu fallen, schliesslich von der Sorge um die finanziellen Folgen der Pflege» [18]. Damit erscheine fraglich, «ob die Urteilsfähigkeit bezüglich des Sterben-Wollens das ausschlaggebende Kriterium für die Bejahung eines autonomen Sterbewunsches sein kann» [18]. Dieser Sichtweise ist grundsätzlich zuzustimmen - jedoch muss betont werden, dass die Urteilsfähigkeit des Patienten oder der Patientin die entscheidende und unverzichtbare Voraussetzung einer Suizidbeihilfe sein muss, wobei die Urteilsfähigkeit in jedem Fall auch noch im Zeitpunkt des Suizids vorzuliegen hat [19]. Neben das Kriterium der Urteilsfähigkeit müssen jedoch weitere klar definierte Kriterien treten, um eine rechtssichere und den ethischen Anforderungen der Suizidbeihilfe gerecht werdende Regelung zu ermöglichen.

Da Ziff. 6.2.1 der Richtlinien die Suizidbeihilfe bei minderjährigen Patientinnen und Patienten nicht ausschliesst und sich der Anwendungsbereich der Richtlinien auch auf Kinder und Jugendliche erstreckt (Ziff. 1 der Richtlinien), stellt sich bei diesen Personen ein ähnliches Abgrenzungsproblem. Was die Frage der Urteilsfähigkeit betrifft, gibt es hier keine feste Altersgrenze - massgebend sind jeweils die Umstände des Einzelfalls [20], so dass Suizidbeihilfe an minderjährigen urteilsfähigen Patienten im Rahmen von Ziff. 6.2.1 der Richtlinien ohne Weiteres möglich wäre. Da Suizid bei Jugendlichen durchaus verbreitet ist [21], wäre es angebracht gewesen, die besondere Notwendigkeit der Suizidprävention bei dieser Personengruppe in der Suizidbeihilferegelung zu berücksichtigen, z.B. indem man Suizidbeihilfe für Minderjährige generell ausgeschlossen bzw. eine entsprechend hohe Altersgrenze festgelegt hätte.

\section{Widersprüche}

Abschliessend sei darauf hingewiesen, dass die Anwendung der Suizidbeihilferegelung oft $\mathrm{zu}$ widersprüchlichen Ergebnissen führen kann. Wie bereits er- 
wähnt, verlangt die Regelung in Bezug auf das Erfordernis des "unerträglichen Leidens» lediglich das Vorliegen bestimmter Krankheitssymptome und Funktionseinschränkungen, weshalb Suizidbeihilfe gestützt auf Ziff. 6.2.1 der Richtlinien auch bei körperlichen Behinderungen, chronischen und sogar heilbaren Krankheiten möglich ist, ohne dass eine bestimmte Diagnose oder Prognose gestellt werden

\section{Die Anwendung der Suizidbeihilferegelung kann oft zu widersprüchlichen Ergebnissen führen.}

muss. Zugleich setzt die Vorschrift aber voraus, dass der Patient leidet und dieses Leiden für ihn unerträglich ist.

Ein Arzt oder eine Ärztin wäre deshalb befugt, bei einem Patienten, der zwar nur leicht erkrankt ist, aber gleichwohl Funktionseinschränkungen oder Krankheitssymptome aufweist, Suizidbeihilfe leisten, sofern nur der Patient behauptet, dass er unter diesen unerträglich leidet. Dies wäre mit den ärztlichen Pflichten, menschliches Leben zu schützen, Krankheiten zu heilen und Leiden zu lindern, insbesondere dann schwer $\mathrm{zu}$ vereinbaren, wenn der Patient an einer heilbaren oder nicht lebensbedrohlichen Krankheit leidet [22]. Bei Patienten, bei denen eine schwere, todbringende Erkrankung diagnostiziert wurde, erlaubt es die neue Regelung demgegenüber nicht, Suizidbeihilfe zu leisten, solange diese keine Symptome oder Funktionseinschränkungen haben, die ein unerträgliches Leiden verursachen. Der Arzt muss vielmehr warten, bis der Patient leidet und dieses Leiden unerträglich geworden ist.

\section{Fazit}

Zusammenfassend lässt sich feststellen, dass das Kriterium des «unerträglichen Leidens» in Ziff. 6.2.1 der Richtlinien zu unbestimmt ist, um eine rechtssichere und vorhersehbare Anwendung der Bestimmung zu gewährleisten. Die Anwendung der neuen Regelung erscheint zudem problematisch bei der Anwendung auf psychisch kranke Patienten - insbesondere im Hinblick auf die Ge- währleistung einer effektiven Suizidprävention - und kann in der Praxis zu widersprüchlichen Ergebnissen führen. Um Rechtssicherheit zu gewährleisten und willkürliche Entscheidungen zu vermeiden, sollte das Kriterium des "unerträglichen Leidens» deshalb durch ein solches ersetzt werden, das klar definiert ist und vom Arzt und gegebenenfalls einer Standeskommission oder einer anderen die Vorschrift anwendenden Instanz problemlos überprüft werden kann. Ausgangspunkt der Regelung sollte deshalb die Diagnose und daran anknüpfend die Prognose sein. Denn diese können vom behandelnden Arzt aufgrund seines Sachverstands mit hinreichender Sicherheit gestellt und überprüft werden. Eine Möglichkeit wäre es etwa, dort anzusetzen, wo bei einer fortschreitenden, tödlichen Erkrankung die Optionen einer kurativen Behandlung des Patienten enden und allenfalls noch eine palliative Behandlung möglich ist.

\section{Literatur}

1 Ebenso Thomas Gächter/Bernhard Rütsche: Gesundheitsrecht, 4. Auflage, Basel 2018, Rz. 62f.

2 Gächter/Rütsche, a.a.O., Rz. 63

3 Gächter/Rütsche, a.a.O., Rz. 64.

4 Siehe insb. BGE 133 I 58 E. 6.3.4ff.

5 Vgl. Stellungnahme der Nationalen Ethikkommission im Bereich Humanmedizin Nr. 13/2006 «Sorgfaltskriterien im Umgang mit Suizidbeihilfe", S. 3.

6 Vgl. z.B. BVGE 2011/53 E. 8.1

7 Vgl. BGE 136 I 87 E. 3.1.

8 BGE 125 I 361 E. 4 a.

9 BGE 136 I 87 E. 3.1.

10 Josef Widler/ Michael Kohlbacher: Suizid bei Krankheit, SÄZ 2018, S. 971

11 Vgl. Widler/Kohlbacher, a.a.O. S. 971 f.

12 Vgl. Widler/Kohlbacher, a.a.O., S. 971.

13 Ziff. 6.2 der Richtlinien.

14 Isabel Baur/Cristina Garcia Gonçalves / Marc Wohlwend: Demenz, Urteilsunfähigkeit und Sterbewunsch, Jusletter vom 27. August 2018, Rz. 7f.

15 Baur/Garcia Gonçalves/Wohlwend, a.a.O., Rz. 8.

16 Vgl. WHO-Bericht «Suizidprävention: Eine globale Herausforderung» (im Internet abrufbar unter http://www.who.int/mental health/suicide-prevention/world_report_2014/en/), S. 42.

17 Vgl. zu dieser Problematik ebenfalls: Widler/Kohlbacher, a.a.O., S. 972.

18 BGE 136 II 415 E. 2.3.4

19 Vgl. Gächter/Rütsche, a.a.O., Rz. 354.

20 BGE 134 II 235 E. 4.3.2 f.

21 Laut WHO-Bericht «Suizidprävention: Eine globale Herausforderung", a.a.O., S. 24, machen Suizide weltweit bei jungen Erwachsenen im Alter von 15 bis 29 Jahren 8,5\% sämtlicher Todesfälle aus und sind die zweithäufigste Todesursache nach Verkehrsunfällen. 22 Vgl. Widler/Kohlbacher, a.a.O., S. 972. 